\title{
Computational Coordinates
}

$\underline{\mathrm{MoO}}\left(\mathrm{NH}_{3}\right)_{2}\left(\mathrm{SCH}_{3}\right)_{2} \mathrm{Cl}$ (1)

$\begin{array}{llll}\text { Mo1 } & 0.385 & 1.013 & 0.257 \\ \text { O2 } & 0.314 & 2.694 & 0.341 \\ \text { N3 } & -1.900 & 0.865 & -0.065 \\ \text { S4 } & 0.257 & 0.730 & 2.639 \\ \text { C15 } & 2.671 & 0.512 & 0.273 \\ \text { S6 } & 0.388 & 0.540 & -2.077 \\ \text { N7 } & -0.155 & -1.435 & 0.531 \\ \text { C8 } & -1.302 & 0.884 & -2.466 \\ \text { C14 } & -0.017 & -0.977 & 2.941 \\ \text { H9 } & -2.127 & 1.309 & -0.814 \\ \text { H20 } & -2.137 & 0.001 & -0.146 \\ \text { H22 } & -2.327 & 1.228 & 0.639 \\ \text { H15 } & -0.929 & -1.524 & 0.980 \\ \text { H21 } & -0.233 & -1.818 & -0.280 \\ \text { H23 } & 0.506 & -1.839 & 0.990 \\ \text { H10 } & -1.735 & 1.144 & -1.721 \\ \text { H11 } & -1.341 & 1.542 & -3.080 \\ \text { H12 } & -1.691 & 0.143 & -2.796 \\ \text { H16 } & -0.793 & -1.082 & 3.385 \\ \text { H17 } & 0.658 & -1.306 & 3.436 \\ \text { H18 } & -0.058 & -1.418 & 2.158\end{array}$

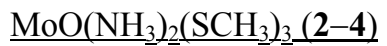

$\begin{array}{llll}\text { Mo1 } & 0.438 & 0.997 & 0.655 \\ \text { O2 } & 0.335 & 2.559 & 1.276 \\ \text { N3 } & -1.812 & 0.624 & 1.039 \\ \text { S4 } & 1.146 & 0.042 & 2.741 \\ \text { S5 } & 2.635 & 0.838 & -0.218 \\ \text { S6 } & -0.342 & 1.203 & -1.586 \\ \text { N7 } & 0.119 & -1.481 & 0.307 \\ \text { C8 } & -2.075 & 1.400 & -1.296 \\ \text { C14 } & 1.058 & -1.702 & 2.567 \\ \text { C24 } & 2.723 & 1.363 & -1.937 \\ \text { H9 } & -2.299 & 1.223 & 0.577 \\ \text { H20 } & -2.028 & -0.208 & 0.772 \\ \text { H22 } & -1.985 & 0.711 & 1.918 \\ \text { H15 } & -0.451 & -1.800 & 0.926 \\ \text { H21 } & -0.218 & -1.628 & -0.515 \\ \text { H23 } & 0.912 & -1.899 & 0.384 \\ \text { H10 } & -2.236 & 1.376 & -0.410 \\ \text { H11 } & -2.347 & 2.191 & -1.628 \\ \text { H12 } & -2.525 & 0.734 & -1.700 \\ \text { H16 } & 0.486 & -2.036 & 3.176 \\ \text { H17 } & 1.874 & -2.058 & 2.695 \\ \text { H18 } & 0.768 & -1.907 & 1.740 \\ \text { H13 } & 1.905 & 1.613 & -2.217 \\ \text { H25 } & 3.290 & 2.058 & -2.010 \\ \text { H29 } & 3.018 & 0.681 & -2.444\end{array}$


$\underline{\mathrm{MoO}}\left(\mathrm{S}_{2} \underline{\mathrm{C}_{2}} \underline{2}\left(\mathrm{CH}_{3}\right)_{2}\right)\left(\mathrm{SCH}_{3}\right)\left(\mathrm{OH}_{2}\right)\left(\mathrm{SO}_{\underline{\mathrm{red}}}\right.$ where $\mathrm{O}=\mathrm{Mo}-\mathrm{S}-\mathrm{C}$ dihedral angle $\left.=80^{\circ}\right)$

$\begin{array}{llll}\text { O1 } & 1.936 & -1.007 & 1.390 \\ \text { C2 } & 4.041 & -0.567 & 0.224 \\ \text { S3 } & 2.777 & 0.100 & -0.860 \\ \text { C4 } & -3.545 & -1.299 & 0.820 \\ \text { C5 } & -2.319 & -0.798 & 0.395 \\ \text { S6 } & -1.106 & -0.881 & 1.518 \\ \text { C7 } & -2.267 & -0.232 & -0.951 \\ \text { S8 } & -0.678 & 0.383 & -1.383 \\ \text { C9 } & -3.526 & -0.192 & -1.928 \\ \text { Mo10 } & 0.732 & 0.262 & 0.518 \\ \text { O11 } & 0.914 & 1.713 & 1.453 \\ \text { H12 } & 1.456 & -1.527 & 2.059 \\ \text { H13 } & 3.797 & -1.586 & 0.558 \\ \text { H14 } & 5.023 & -0.618 & -0.284 \\ \text { H15 } & 4.162 & 0.047 & 1.124 \\ \text { H19 } & -3.473 & -2.324 & 1.243 \\ \text { H20 } & -4.002 & -0.713 & 1.648 \\ \text { H21 } & -4.320 & -1.355 & 0.043 \\ \text { H16 } & -3.189 & 0.200 & -2.895 \\ \text { H17 } & -3.957 & -1.190 & -2.090 \\ \text { H18 } & -4.322 & 0.467 & -1.548\end{array}$

$\underline{\mathrm{MoO}}\left(\mathrm{S}_{2} \underline{\mathrm{C}}_{2}\left(\mathrm{CH}_{3}\right)_{2}\right)\left(\mathrm{SCH}_{3}\right)\left(\mathrm{OH}_{2}\right)\left(\mathrm{SO}_{\text {red }}\right.$ where $\mathrm{O}=\mathrm{Mo}-\mathrm{S}-\mathrm{C}$ dihedral angle $\left.=180^{\circ}\right)$

$\begin{array}{llll}\text { O1 } & 1.893 & -1.761 & 0.099 \\ \text { C2 } & 2.687 & 0.563 & -2.117 \\ \text { S3 } & 2.808 & 0.683 & -0.331 \\ \text { C4 } & -3.554 & -1.352 & -0.681 \\ \text { C5 } & -2.320 & -0.740 & -0.489 \\ \text { S6 } & -1.153 & -1.716 & 0.162 \\ \text { C7 } & -2.222 & 0.675 & -0.839 \\ \text { S8 } & -0.626 & 1.352 & -0.547 \\ \text { C9 } & -3.440 & 1.510 & -1.439 \\ \text { Mo10 } & 0.706 & -0.275 & 0.547 \\ \text { O11 } & 0.826 & -0.157 & 2.275 \\ \text { H12 } & 1.394 & -2.597 & 0.065 \\ \text { H13 } & 1.864 & 1.175 & -2.513 \\ \text { H14 } & 3.617 & 0.908 & -2.609 \\ \text { H15 } & 2.504 & -0.470 & -2.438 \\ \text { H19 } & -3.484 & -2.306 & -1.245 \\ \text { H20 } & -4.055 & -1.652 & 0.267 \\ \text { H21 } & -4.296 & -0.745 & -1.220 \\ \text { H16 } & -3.070 & 2.510 & -1.693 \\ \text { H17 } & -3.848 & 1.051 & -2.351 \\ \text { H18 } & -4.261 & 1.619 & -0.715\end{array}$




\begin{tabular}{llll}
$\mathrm{MoOS}\left(\mathrm{S}_{2}\right.$ & $\mathrm{C}_{2}$ \\
\hline $\mathrm{Ho}$ & $\left.\frac{\mathrm{H}_{2}}{2}\right)\left(\mathrm{OH}_{2}\right)\left(\mathrm{XO}_{\mathrm{ox}}\right)$ \\
$\mathrm{O} 2$ & 1.707 & 0.455 & 0.561 \\
$\mathrm{~S} 3$ & 1.701 & 0.448 & 2.261 \\
$\mathrm{~S} 4$ & 0.175 & 2.062 & -0.285 \\
$\mathrm{O} 5$ & 2.159 & -1.130 & -0.298 \\
$\mathrm{~S} 6$ & 3.416 & -0.889 & 0.110 \\
$\mathrm{C} 7$ & -1.192 & 1.193 & -0.002 \\
$\mathrm{C} 8$ & -1.199 & -0.248 & -0.840 \\
$\mathrm{C} 10$ & -2.397 & 1.940 & -1.339 \\
$\mathrm{C} 11$ & -2.421 & -0.973 & -1.327 \\
$\mathrm{H} 9$ & 3.522 & -1.479 & 0.702 \\
$\mathrm{H} 12$ & 3.279 & -1.208 & -0.787 \\
$\mathrm{H} 18$ & -3.095 & 1.285 & -1.631 \\
$\mathrm{H} 19$ & -2.763 & 2.513 & -0.606 \\
$\mathrm{H} 20$ & -2.136 & 2.516 & -2.114 \\
$\mathrm{H} 15$ & -2.606 & -0.721 & -2.277 \\
$\mathrm{H} 16$ & -2.267 & -1.960 & -1.269 \\
$\mathrm{H} 17$ & -3.203 & -0.724 & -0.757
\end{tabular}




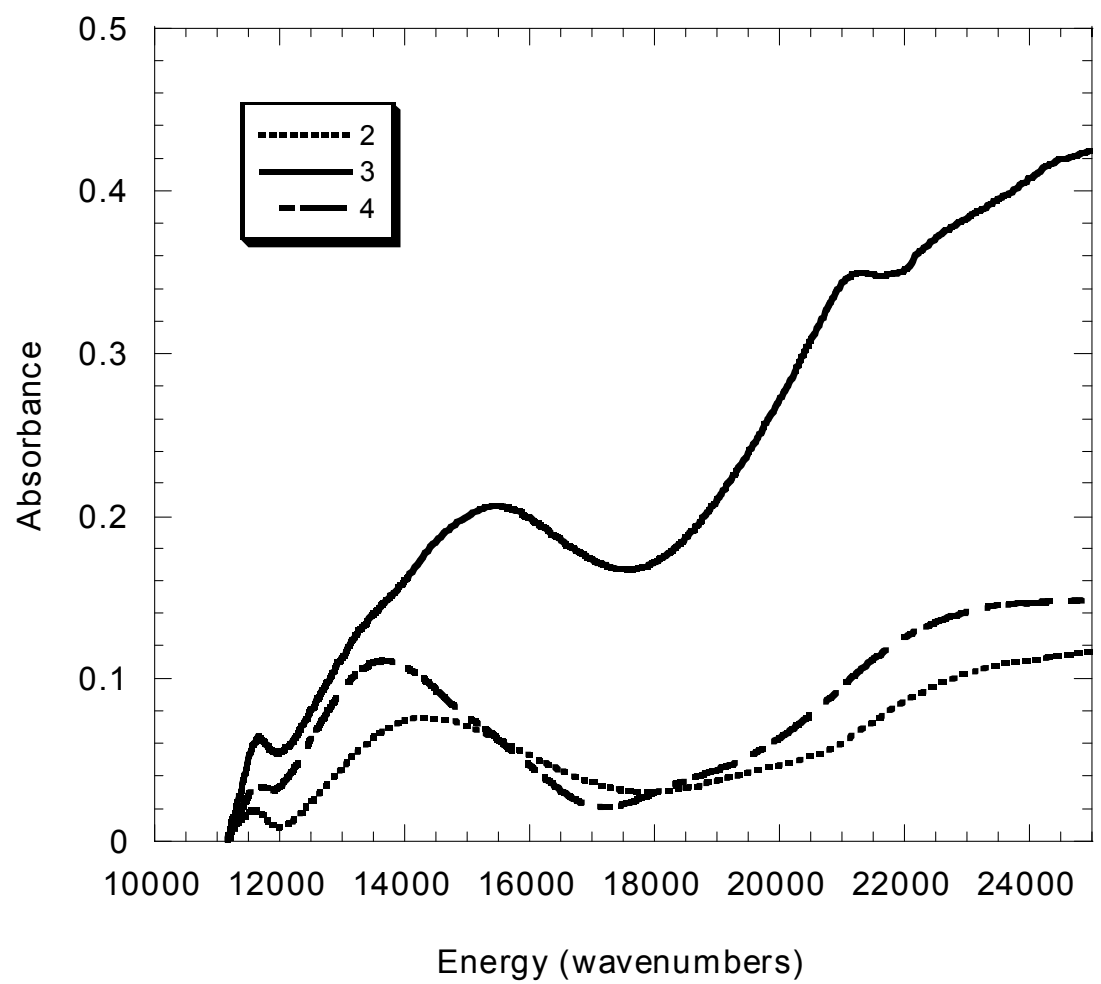

Figure S1. Room temperature PDMS mull absorption spectra of $\mathbf{2 , 3}$, and $\mathbf{4}$

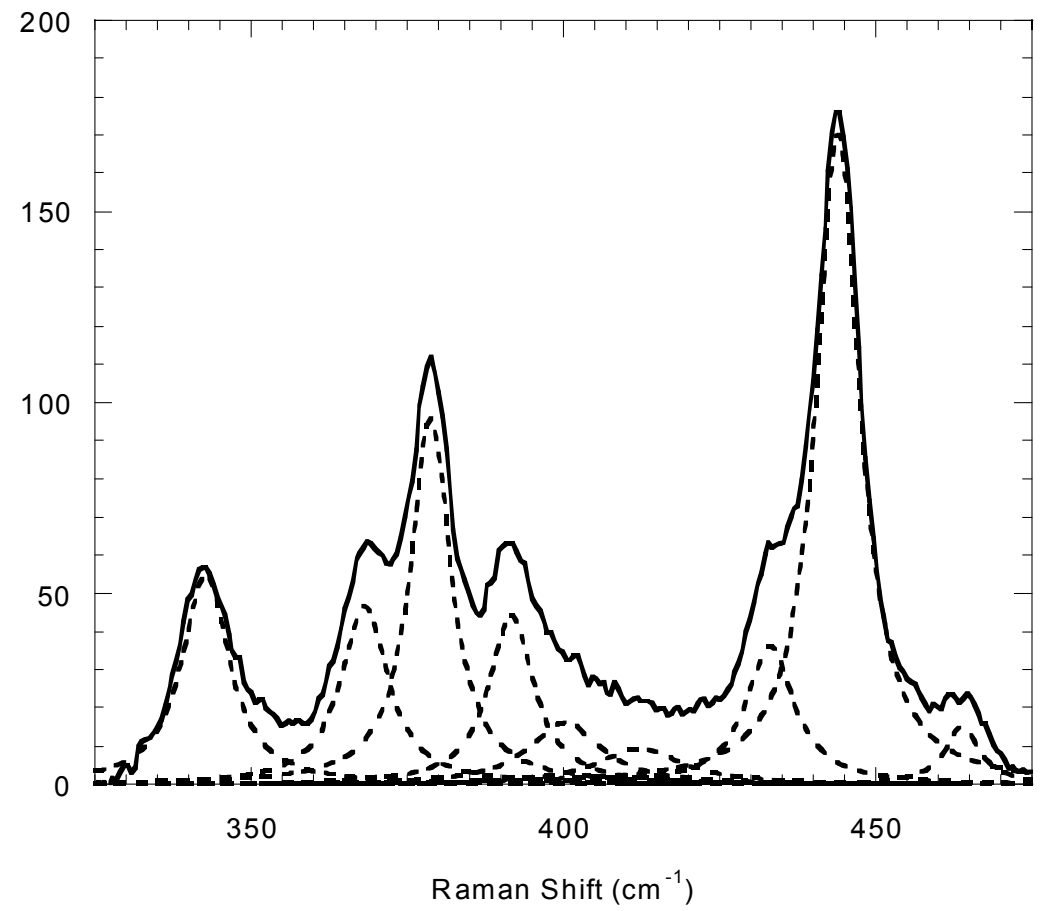

Figure S2. Deconvoluted room temperature resonance Raman spectrum of complex 4 

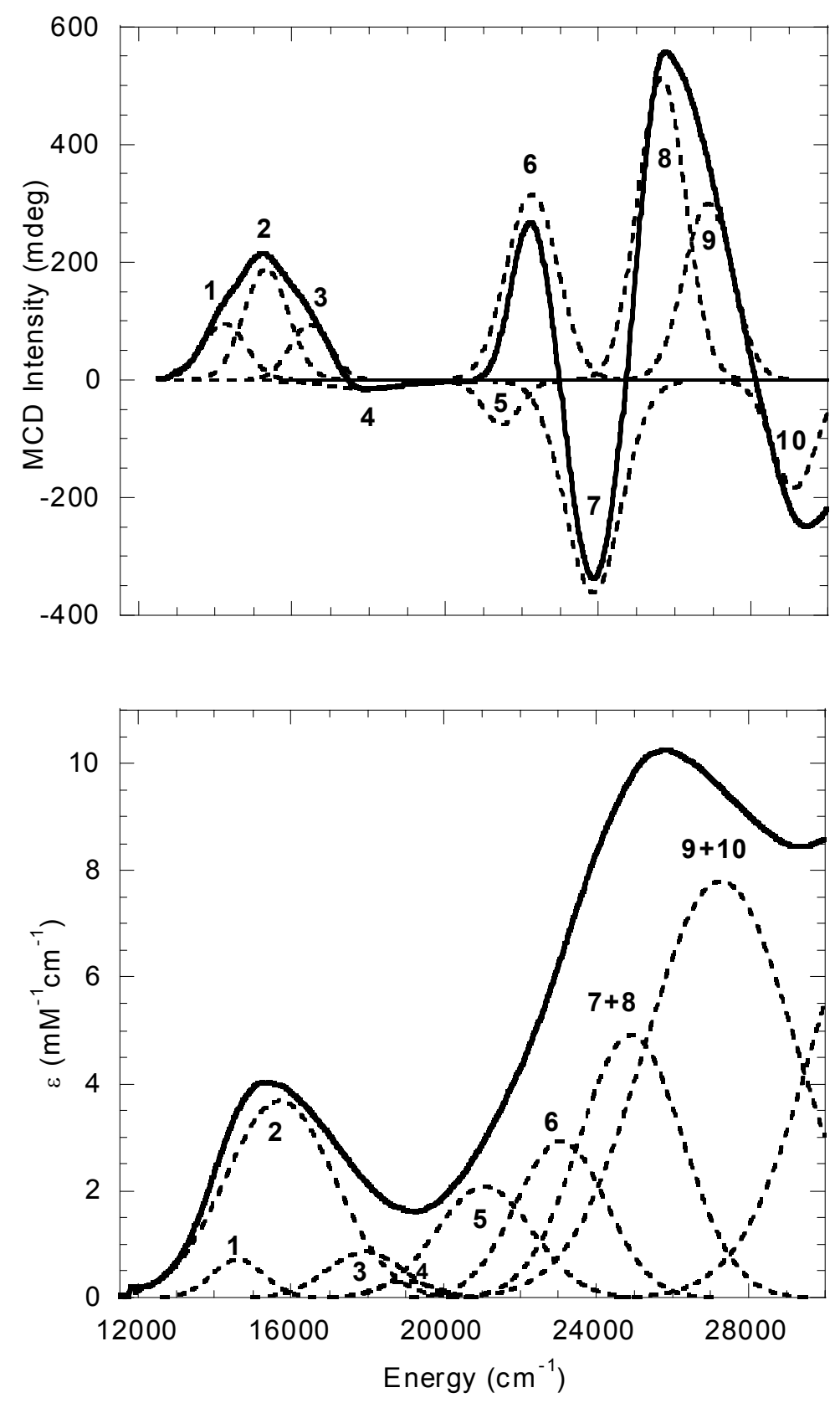

Figure S3. Gaussian resolved (top) MCD spectra and (bottom) electronic absorbance spectra of complex 2 

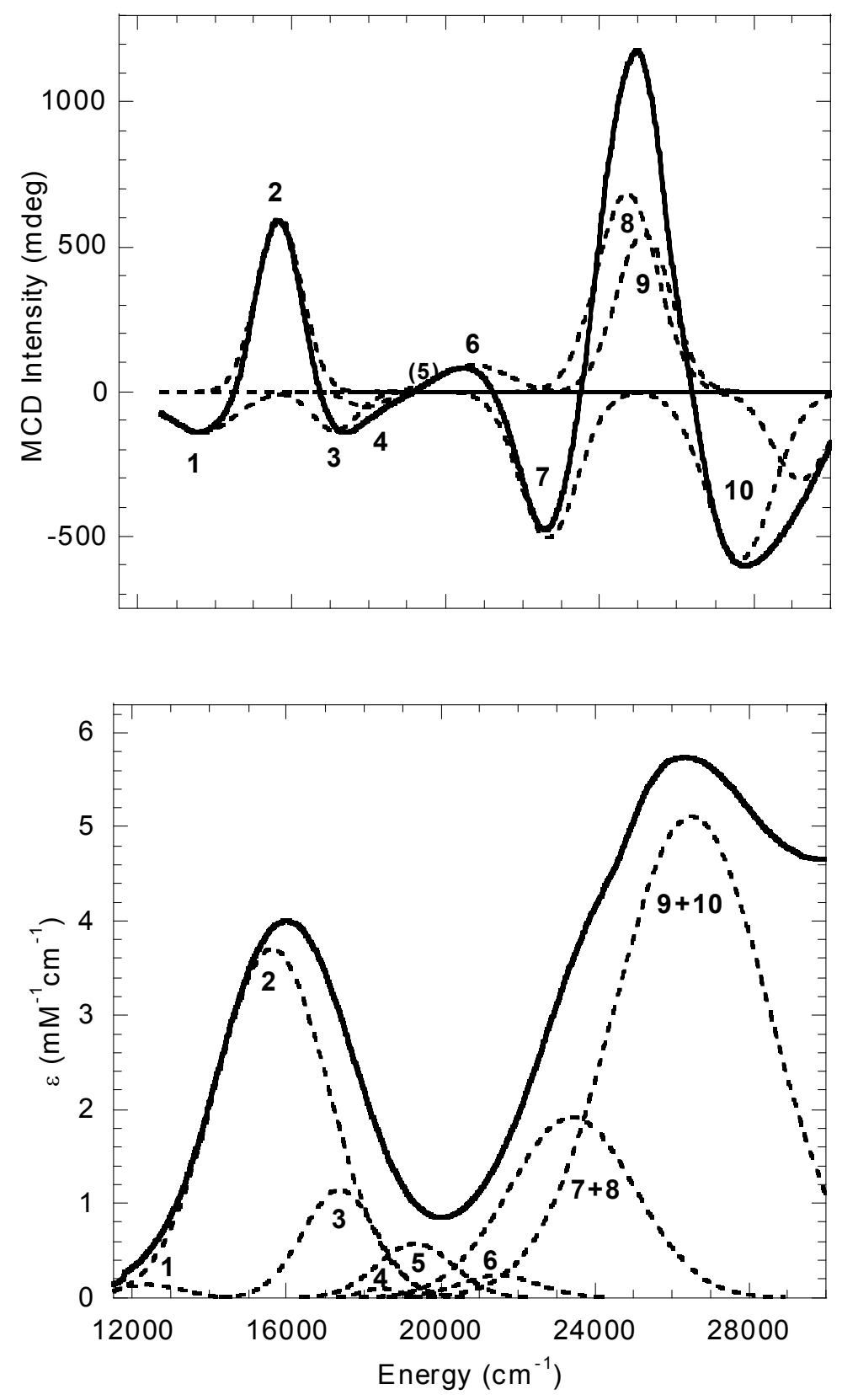

Figure S4. Gaussian resolved (top) MCD spectra and (bottom) electronic absorbance spectra of complex 3 


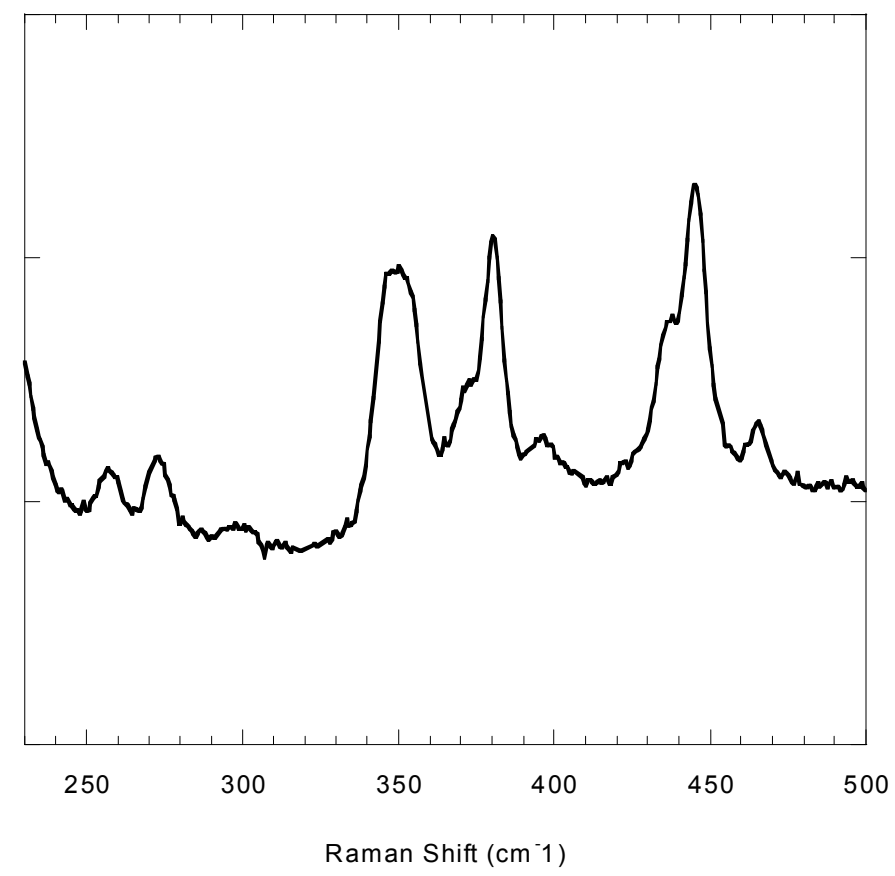

Figure S5. Room temperature resonance Raman spectra of $\left(\mathrm{L}-N_{2} S_{2}\right) \mathrm{MoO}(\mathrm{SePh})$ 